\title{
Low Platelet Level May be a Predictor for Mortality in Adult Patients with Common Variable Immune Deficiency
}

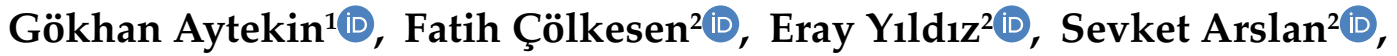 \\ Ahmet Zafer Çalışkaner ${ }^{2}$ (D) \\ 1 Konya City Hospital, Division of Allergy and Clinical Immunology, Konya, Turkey \\ 2 Necmettin Erbakan University, Meram Faculty of Medicine, Department of Internal Medicine, Division of Allergy and Clinical \\ Immunology, Konya, Turkey
}

\begin{abstract}
Background: Common variable immunodeficiency (CVID) is the most common symptomatic immunodeficiency in adults. We, therefore, aimed to reveal mortality rates, causes of mortality in CVID patients, as well as demographic and clinical characteristics and differences of survived and dead CVID patients

Methods: The study group included 50 patients [(Female: $23(46 \%)$, Male: $27(54 \%)$ ] with CVID, who were followed up on a regular basis for a period of ten years (115.18 \pm 80.74 months).

Results: Diagnostic delay was 84 (0-360) months, and the mean follow-up time was $115.18 \pm 80.74$ months. The most common clinical presenting complaints were frequent and recurrent infections and pneumonia. At diagnosis, serum IgG levels were 1.72 $(0.33-6.90) \mathrm{g} / \mathrm{L}$. The overall survival rate of the patients during the follow-up time was $88 \%$. As a result of univariate Cox regression analysis, platelet count was determined to be an independent risk factor for mortality in CVID patients (Hazard ratio, HR: $0.990,95 \%$ confidence interval, CI: 981-0.999, p: 0.025). When the patients were classified according to mean platelet counts (platelets $<207770 / \mathrm{mm} 3$ and platelets $>207770 / \mathrm{mm} 3$ ), the mortality rate in the patient group with platelets $<207770 / \mathrm{mm} 3 \mathrm{was}$ determined to be statistically significantly higher compared to the patient group with platelets > 207770/mm3 (log-rank: 0.013).

Conclusions: Clinicians dealing with this patient group should remember that immune dysregulation and low platelet count are independent risk factors for mortality and they should remarkably follow up patients with low platelet count closely.
\end{abstract}

Keywords: Platelets, Common Variable Immune Deficiency, Mortality.

Cite this article as: Aytekin G, Çölkesen F, Ylldız E, Arslan Ş, Çalş̧kaner AZ. Low platelet level may be a predictor for mortality in adult patients with Common Variable Immune Deficiency. Arch Curr Med Res. 2021;2(3):153-160 


\section{INTRODUCTION}

Common variable immunodeficiency (CVID) is the most common symptomatic immunodeficiency in adults (1). The expression "variable" included in the name defines the heterogeneity of manifestations. The patients suffer from frequent and recurrent infections and disorders affecting several organs and systems, including chronic pulmonary disease, immune dysregulation, autoimmunity, lymphoproliferation, granulomatous diseases, and tendency to malignancies. Diagnosis of CVID is established by low levels of serum IgG together with low levels of IgM and / or IgA, poor vaccine response, and exclusion of secondary causes that may lead to immunodeficiency $(2,3)$. Its prevalence is considered to be between 1:25.000 and 1:50.000 (4). Although CVID may develop at any age, its findings peak at childhood and early adulthood (5). Due to insufficient awareness of physicians on this issue and reasons such as consideration of primary immunodeficiencies as childhood diseases, the diagnostic delay is, unfortunately, common (6). CVID patients have increased mortality compared to the normal population because of infectious and non-infectious complications (7). In several studies, CVID-related mortality has been shown to vary between $15 \%$ to $29 \%(5,7,8)$. Owing to the reduction of mortality caused by infections with immunoglobulin replacement therapy (IGRT), chronic complications such as chronic pulmonary diseases and malignancies have become significant causes of mortality (9). In previous studies, lymphoid malignancies and autoimmunity have been independent risk factor for mortality and emphasized that autoimmune cytopenia is associated with increased prevalence of bronchiectasis, morbidity, and mortality $(10,11)$. Thus, the demonstration of risk factors and causes of mortality in this patient group is crucial for both diagnosis of the patients and the treatment of complications.

Therefore, we aimed to reveal mortality rates, causes of mortality in CVID patients who were being followed-up in our clinic, demographic and clinical characteristics and differences of survived and dead CVID patients, and the effect of platelet counts on mortality in these patients.

\section{MATERIALS AND METHODS}

The study group included 50 patients with CVID who were followed up on a regular basis for a period of ten years (115.18 \pm 80.74 months). Diagnosis of CVID was made according to updated diagnostic criteria of ESID (European Society for Immunodeficiencies) (3). In that cross-sectional study, patient records of who were being followed up with a diagnosis of CVID between 2010 and 2020 were reviewed. Patients' demographic data (including current age, gender, age at diagnosis, diagnostic delay, consanguinity, smoking status, type of immunoglobulin replacement therapy, the status of prophylactic antibiotic use) and clinical characteristics at diagnosis (presence of severe lymphopenia, presence of bronchiectasis, presence of splenomegaly, spirometry results, complete blood count parameters, serum immunoglobulin levels, and peripheral lymphocyte subsets) were obtained from their files. The lymphocyte count $<1000$ cells $/ \mathrm{mm}^{3}$ was considered severe lymphopenia.

Complete blood count was measured with Sheath reagent by Abbott Cell Dyn 3700 series (Chicago, USA). Quantitative determination of serum $\operatorname{IgG}, \operatorname{IgM}$, $\operatorname{Ig} A$, and $\operatorname{IgE}$ was made by means of particle-enhanced immunonephelometry using the Siemens BN II/ BN ProSpec system (Erlangen, Germany). Peripheral blood lymphocyte subsets were measured using the BD FACS Canto II 8-color configuration flow cytometer system (New Jersey, USA) with fluorescently labeled antibodies.

Spirometric measurements were obtained using a common protocol with the nSpire ZAN 100 spirometer. Three maneuvers were performed, although additional tests may be needed if one or more of the curves are unacceptable. The forced expiratory volume in one second (FEV1), the ratio of FEV1/FVC (forced vital capacity), peak expiratory flow (PEF), and mean expiratory flow $25 \%-75 \%$ of predicted values for similar age, sex, race, and height were recorded.

The study protocol was approved by the Necmettin Erbakan University Meram Medical Faculty Ethics Committee (Date: 03.04.2020 - No: 2020 / 2401). This study was performed according to the ethical standards laid down Declaration of Helsinki and its later amendments. The authors carried out no animal or human studies for this article.

Statistical analysis was performed with IBM SPSS Statistics Version 22 software package. Normally distributed parameters were presented as mean \pm standard deviation, and data that is not normally distributed were expressed as median (minimum-maximum). While Pearson correlation analysis was used for normally distributed parameters, 
Spearman rank correlation analysis was used for nonparametric variables. Descriptive data were presented as frequencies and percentages and compared using the Chisquare test. Comparisons between baseline characteristics were performed by independent Student $t$, MannWhitney rank-sum, Fisher exacts, or Chi-square tests where appropriate. Binomial logistic regression analysis was performed to determine independent predictors for mortality. To determine independent predictors for mortality Cox regression analysis and Kaplan Meiers test were performed.

\section{RESULTS}

Fifty CVID patients [(Female: 23 (46\%), Male: 27 (54\%)] were included in the study. Of the study population, the median age was 37 (23-67) years, and the age at diagnosis was $27.94 \pm 13.64$ years. The diagnostic delay was 84 (0360) months, and the mean follow-up time was 115.18 \pm 80.74 months. The most common clinical presenting complaints were frequent and recurrent infections and pneumonia.

Of the patients, 30\% had severe lymphopenia, 56\% splenomegaly, and $60 \%$ bronchiectasis. At diagnosis, serum IgG levels were $1.72(0.33-6.90) \mathrm{g} / \mathrm{L}$ and switched memory B cell percentages $1.70(0-52.0)$. The overall survival rate of the patients during the follow-up time was $88 \%$. The Demographic, clinical, and laboratory characteristics of the study population were summarized in Table 1.

Table 1. Baseline demographic, clinical, and immunological parameters of the study population

\begin{tabular}{|c|c|c|c|}
\hline \multicolumn{2}{|l|}{ Demographic properties } & \multicolumn{2}{|l|}{ Clinical and Immunological parameters } \\
\hline Gender, Female, n (\%) & $23(46)$ & Neutrophil count, $\mathrm{mm}^{3}$ & $3660(1000-12500)$ \\
\hline Current age, year & $37(23-67)$ & Lymphocyte, $\mathrm{mm}^{3}$ & $1445(400-8900)$ \\
\hline Age at diagnosis, year & $27.94 \pm 13.64$ & Platelet count, $\mathrm{mm}^{3}$ & $207770 \pm 96657$ \\
\hline Diagnostic delay, month & $84(0-360)$ & Neutrophil / lymphocyte ratio & $2.45(0.74-7.50)$ \\
\hline Follow-up time, month & $115.18 \pm 80.74$ & Platelet / lymphocyte ratio & $114.82(9.08-607)$ \\
\hline Consanguinity, n (\%) & $22(44)$ & $\mathrm{IgG}, \mathrm{g} / \mathrm{L}$ & $1.72(0.33-6.90)$ \\
\hline Smoking, n (\%) & $7(14)$ & $\operatorname{IgM}, g / L$ & $0.26(0.06-5.99)$ \\
\hline SCIG, n (\%) & $14(28)$ & $\operatorname{IgA}, \mathrm{g} / \mathrm{L}$ & $0.24(0-190)$ \\
\hline Severe lymphopenia, n (\%) & $15(30)$ & $\operatorname{IgE}, \mathrm{g} / \mathrm{L}$ & $17(5-220)$ \\
\hline Bronchiectasis, n (\%) & $30(60)$ & $\mathrm{CD}^{+} \mathrm{T}$ cells, $\%$ & $76.54 \pm 11.47$ \\
\hline Low FEV1, n (\%) & $25(50)$ & $\mathrm{CD}^{+} \mathrm{T}$ cells, $\%$ & $31.66 \pm 14.32$ \\
\hline Malignancy, n (\%) & $3(6)$ & $\mathrm{CD}^{+} \mathrm{T}$ cells, $\%$ & $37.50(19-74)$ \\
\hline Prophylaxis, n (\%) & $38(76)$ & CD19 ${ }^{+}$B cells, $\%$ & $7.10 \pm 5.95$ \\
\hline BMI & $25.75(14.80-49.0)$ & CD16 ${ }^{+}-56^{+}$NK cells, $\%$ & $7.50(0-26.0)$ \\
\hline FEV1 & $72.17 \pm 17.68$ & IgM- CD27+ Switched Memory B cells, $\%$ & $1.70(0-52.0)$ \\
\hline FEV1 / FVC & $98.02 \pm 10.12$ & IgM+ CD27- Naive B cells, $\%$ & $84.55(0-98.60)$ \\
\hline PEF & $66.50(3.80-100)$ & Mortality & $6(12)$ \\
\hline MEF25-75 & $59.53 \pm 25.15$ & Splenomegaly, n (\%) & $28(56)$ \\
\hline
\end{tabular}

CVID: Common variable immune deficiency, Ig: immune globulin, SCIG: subcutaneous immune globulin, CD: The cluster of differentiation, FEV1: The forced expiratory volume in 1 second, FVC: forced vital capacity, PEF: The peak expiratory flow, MEF25-75: the mean expiratory flow between the 25\% and 75\% of the FVC 
When the patients who survived and died during followup time were compared, there was no significant difference between the groups in regard to gender, duration of diagnostic delay, serum IgG level at diagnosis, CD19+ B cell and IgM- CD27+ Switched Memory B cell percentages, as well as the presence of severe lymphopenia, splenomegaly, bronchiectasis, and malignancy. There was a statistically significant difference in regard to the current age, age at diagnosis, IgM levels at diagnosis, platelet counts and platelet/lymphocyte ratio (p: 0.039, p: 0.049, p: 0.041, p: 0.011, p: 0.042 and p: 0.019, respectively). A comparison of demographic, laboratory and immunological parameters of survived and dead CVID patients was summarized in Table 2.

Table 2. Comparison of demographic, clinical and laboratory parameters of CVID patients who died and survived

\begin{tabular}{|c|c|c|c|}
\hline & Dead $(\mathrm{N}=6)$ & Alive (N: 44) & $\mathrm{p}$ \\
\hline Gender, F, n (\%) & $1(16.7)$ & $22(50)$ & 0.124 \\
\hline Current age, years & $61(59-63)$ & $36.5(23-67)$ & 0.039 \\
\hline Age at diagnosis & $38.17 \pm 17.31$ & $26.55 \pm 12.67$ & 0.049 \\
\hline Diagnostic delay, month & $48(0-120)$ & $84(0-360)$ & 0.211 \\
\hline Follow up time, month & $127.17 \pm 83.08$ & $105.09 \pm 94.33$ & 0.702 \\
\hline Consanguinity, n (\%) & $2(33.3)$ & $20(45.5)$ & 0.746 \\
\hline Smoking, $\mathrm{n}(\%)$ & 0 & $7(16.3)$ & 0.329 \\
\hline SCIG, n (\%) & 0 & $14(31.8)$ & 0.103 \\
\hline Severe lymphopenia, $\mathrm{n}(\%)$ & $2(33.3)$ & $13(29.5)$ & 0.849 \\
\hline Splenomegaly, n (\%) & $4(66.7)$ & $24(54.5)$ & 0.575 \\
\hline Bronchiectasis, n (\%) & $5(83.3)$ & $25(56.8)$ & 0.214 \\
\hline Malignancy, n (\%) & 0 & $3(6.8)$ & 0.509 \\
\hline Prophylaxis, n (\%) & $4(66.7)$ & $34(77.3)$ & 0.568 \\
\hline BMI & $28.6(28.19-29)$ & $24.68(14.80-49)$ & 0.151 \\
\hline Neutrophil count, $\mathrm{mm}^{3}$ & $1450(1000-1900)$ & $3825(1058-12500)$ & 0.388 \\
\hline Lymphocyte count, $\mathrm{mm}^{3}$ & $1100(1000-1200)$ & $1520(400-8900)$ & 0.404 \\
\hline Platelet count, $\mathrm{mm}^{3}$ & $115333 \pm 103511$ & $220375 \pm 89697$ & 0.011 \\
\hline Neutrophil / Lymphocyte ratio & $1290(1.0-1.58)$ & $2590(0.74-7.50)$ & 0.439 \\
\hline Platelet / lymphocyte ratio & $51.92(50.83-53.0)$ & $119.15(9.08-607)$ & 0.042 \\
\hline FEV1, $\%$ & $61.33 \pm 15.28$ & $73.04 \pm 17.75$ & 0.275 \\
\hline FEV1/FVC & $97.67 \pm 13.61$ & $98.05 \pm 10.03$ & 0.951 \\
\hline PEF & $42.4(3.80-81.0)$ & $66.5(5.39-100.0)$ & 0.401 \\
\hline MEF 25/75 & $67.33 \pm 28.50$ & $59.01 \pm 25.19$ & 0.588 \\
\hline IgG at diagnosis, $\mathrm{g} / \mathrm{L}$ & $1.96(1.17-2.75)$ & $1.72(0.33-6.90)$ & 0.886 \\
\hline IgM at diagnosis, $\mathrm{g} / \mathrm{L}$ & $0.26(0.09-0.44)$ & $0.26(0.06-5.99)$ & 0.041 \\
\hline IgA at diagnosis, $\mathrm{g} / \mathrm{L}$ & $0.09(0.07-0.11)$ & $0.25(0-1.90)$ & 0.116 \\
\hline IgE at diagnosis, IU / mL & $5(5-5)$ & $17.25(5-220)$ & 0.568 \\
\hline $\mathrm{CD}^{+} \mathrm{T}$ cells, $\%$ & $78.60 \pm 7.34$ & $76.31 \pm 11.89$ & 0.677 \\
\hline $\mathrm{CD}^{+}{ }^{\mathrm{T}}$ cells, $\%$ & $28.00 \pm 24.63$ & $32.08 \pm 13.07$ & 0.552 \\
\hline $\mathrm{CD}^{+} \mathrm{T}$ cells, $\%$ & $31(19-43)$ & $37.5(19-74)$ & 0.357 \\
\hline $\mathrm{CD} 19^{+} \mathrm{B}$ cells, $\%$ & $7.60 \pm 7.63$ & $7.05 \pm 5.84$ & 0.846 \\
\hline CD16 $-56^{+}$NK cells, $\%$ & $11(7-15)$ & $7.50(0-26)$ & 0.449 \\
\hline IgM- CD27+ Switched Memory B cells, $\%$ & $0.05(0-0.10)$ & $2.20(0-52.0)$ & 0.472 \\
\hline IgM $^{+}$CD27- Naive B cells, $\%$ & $93.20(90-96.40)$ & $83.50(0-98.60)$ & 0.256 \\
\hline
\end{tabular}

CVID: Common variable immune deficiency, Ig: immune globulin, SCIG: subcutaneous immune globulin, BMI: Body mass index, CD: The cluster of differentiation, FEV1: The forced expiratory volume in 1. second, FVC: forced vital capacity, PEF: The peak expiratory flow, MEF25-75: the mean expiratory flow between the $25 \%$ and $75 \%$ of the FVC, CD: The cluster of differentiation 
As a result of univariate Cox regression analysis, platelet count was determined to be an independent risk factor for mortality in CVID patients (Hazard ratio, HR: 0.990, 95\% confidence interval, CI: 981-0.999, p: 0.025) (Table 3 and Table 4). As a result of multivariate Cox regression analysis; current age, BMI, and neutrophil/lymphocyte ratio were not found to be independent predictors for mortality, whereas platelet count was determined to be an independent predictor for mortality (HR: 0.990, CI:
0.981-0.999, p: 0.025) (Table 5). When the patients were classified according to mean platelet counts (platelets < $207770 / \mathrm{mm}^{3}$ and platelets $>207770 / \mathrm{mm}^{3}$ ), the mortality rate in the patient group with platelets $<207770 / \mathrm{mm}^{3}$ was determined to be statistically significantly higher compared to the patient group with platelets $>207770$ / $\mathrm{mm}^{3}$ (log-rank: 0.013) (Figure 1). The most common causes of mortality of the patients were pneumonia and pneumonia-induced sepsis (Figure 2).

Table 3. Univariate Cox regression analyses demonstrating the relationship between demographic characteristics and mortality in CVID patients

\begin{tabular}{|l|l|l|}
\hline Variables & Univariate Analysis & \\
\hline Gender & HR $(95 \%$ CI $)$ & P value \\
\hline Age & $3.527(0.410-30.323)$ & 0.251 \\
\hline Diagnostic delay & $1.043(0.983-1.106)$ & 0.165 \\
\hline Consanguinity & $0.992(0.977-1.007)$ & 0.308 \\
\hline Smoking & $1.418(0.446-4.321)$ & 0.539 \\
\hline BMI & $0.039(0-2817.666)$ & 0.571 \\
\hline Severe lymphopenia & $0.902(0.773-1.054)$ & 0.194 \\
\hline Splenomegaly & $1.672(0.302-9.261)$ & 0.556 \\
\hline Bronchiectasis & $0.900(0.378-2.147)$ & 0.813 \\
\hline Malignancy & $2.307(0.266-20.021)$ & 0.448 \\
\hline Prophylaxis & $4.87(0.009-2761.254)$ & 0.624 \\
\hline CVID: Con & 0.230 \\
\hline
\end{tabular}

CVID: Common variable immune deficiency, BMI: Body Mass Index

Table 4. Univariate Cox regression analyses demonstrating the relationship between laboratory and immunological parameters and mortality in CVID patients

\begin{tabular}{|c|c|c|}
\hline \multirow[t]{2}{*}{ Variables } & \multicolumn{2}{|l|}{ Univariate Analysis } \\
\hline & $\mathrm{HR}(95 \% \mathrm{CI})$ & P value \\
\hline Neutrophil count & $1.000(1.000-1.000)$ & 0.451 \\
\hline Lymphocyte count & $1.000(0.999-1.000)$ & 0.379 \\
\hline Platelet count $\left(\times 10^{3}\right)$ & $0.990(0.981-0.999)$ & 0.025 \\
\hline Neutrophil/ lymphocyte ratio & $1.059(0.988-1.136)$ & 0.106 \\
\hline Platelet/ lymphocyte ratio & $1.002(0.995-1.008)$ & 0.619 \\
\hline FEV1 & $0.969(0.916-1.024)$ & 0.267 \\
\hline FEV1 / FVC & $1.007(0.889-1.140)$ & 0.918 \\
\hline IgG level, at diagnosis & $0.926(0.587-1.461)$ & 0.741 \\
\hline IgM level, at diagnosis & $0.119(0-70.426)$ & 0.513 \\
\hline IgA level, at diagnosis & $0.023(0-19.171)$ & 0.272 \\
\hline IgE level, at diagnosis & $0.969(0.859-1.094)$ & 0.615 \\
\hline $\mathrm{CD}^{+} \mathrm{T}$ cells, $\%$ & $1.015(0.940-1.096)$ & 0.700 \\
\hline $\mathrm{CD} 4^{+} \mathrm{T}$ cells, $\%$ & $0.982(0.902-1.068)$ & 0.688 \\
\hline $\mathrm{CD}^{+} \mathrm{T}$ cells, $\%$ & $1.010(0.949-1.076)$ & 0.749 \\
\hline $\mathrm{CD}_{19}{ }^{+} \mathrm{B}$ cells, $\%$ & $1.020(0.883-1.178)$ & 0.786 \\
\hline CD16 ${ }^{+}-56^{+}$NK cells, $\%$ & $0.933(0.816-1.067)$ & 0.313 \\
\hline IgM- CD27 $^{+}$Switched Memory B cells, $\%$ & $0.982(0.866-1.115)$ & 0.784 \\
\hline $\mathrm{IgM}^{+}$CD27- Naive B cells, $\%$ & $1.127(0.861-1.475)$ & 0.383 \\
\hline
\end{tabular}

CVID: Common variable immune deficiency, BMI: Body Mass Index, Ig: immune globulin, SCIG: subcutaneous immune globulin, CD: The cluster of differentiation, FEV1: The forced expiratory volume in 1 second, FVC: forced vital capacity, PEF: The peak expiratory flow, MEF25-75: the mean expiratory flow between the $25 \%$ and $75 \%$ of the FVC 
Table 5. Multivariate Cox regression analyses demonstrating the relationship between baseline characteristics and mortality in CVID patients

\begin{tabular}{|l|l|l|}
\hline Variables & Multivariate Analysis \\
\hline & HR $(95 \%$ CI $)$ & P value \\
\hline Age & $1.013(0.948-1.083)$ & 0.706 \\
\hline BMI & $0.898(0.714-1.130)$ & 0.360 \\
\hline Platelet count $\left(\times 10^{3}\right)$ & $0.990(0.981-0.999)$ & $\mathbf{0 . 0 2 5}$ \\
\hline Neutrophil/ lymphocyte ratio & $0.973(0.880-1.076$ & 0.594 \\
\hline
\end{tabular}

CVID: Common variable immune deficiency, BMI: Body Mass Index

Figure 1. The mortality rate in the patient groups with platelets $<207770 / \mathrm{mm} 3$ and platelets $>207770 / \mathrm{mm} 3$

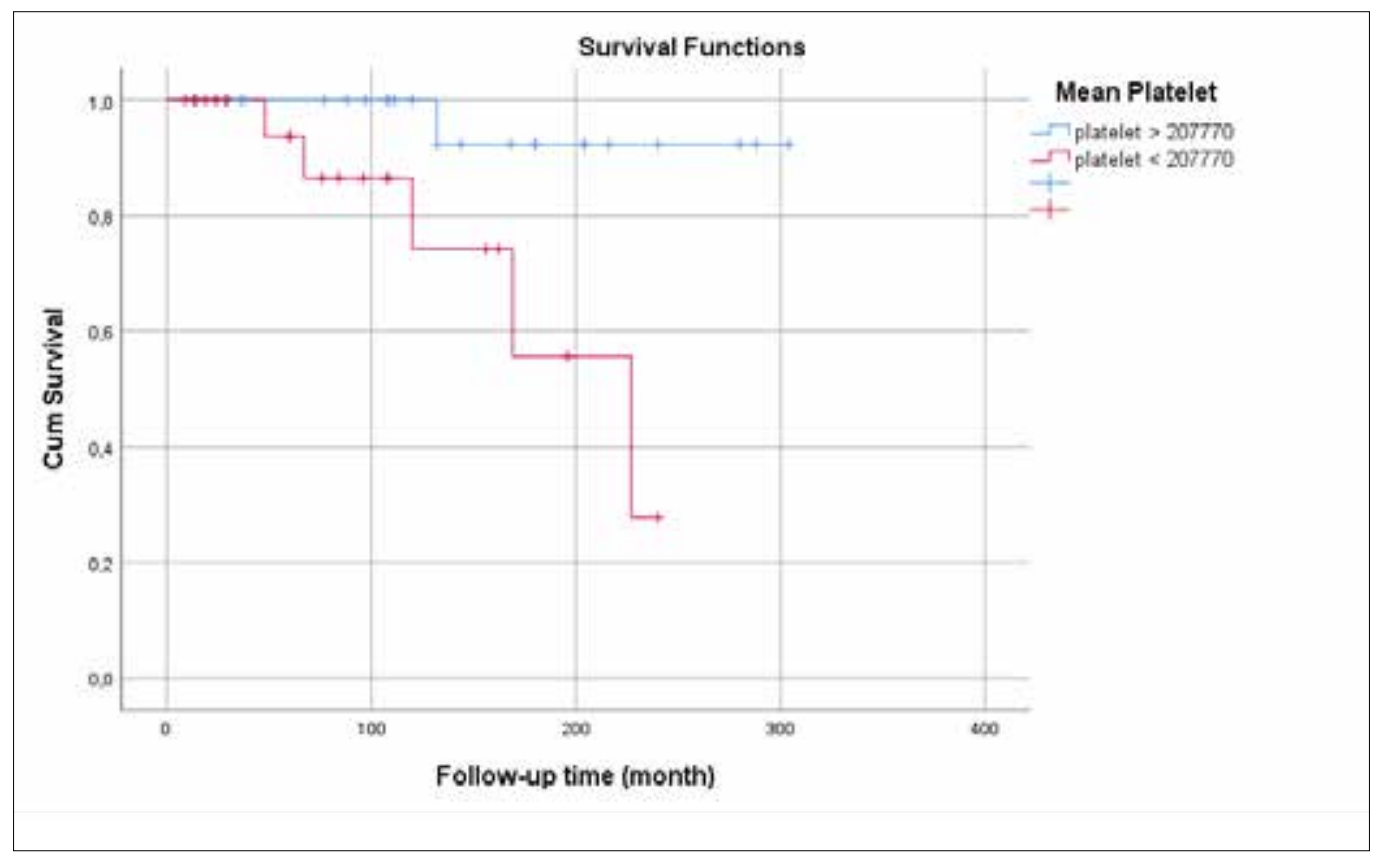

Figure 2. The predominant causes of death

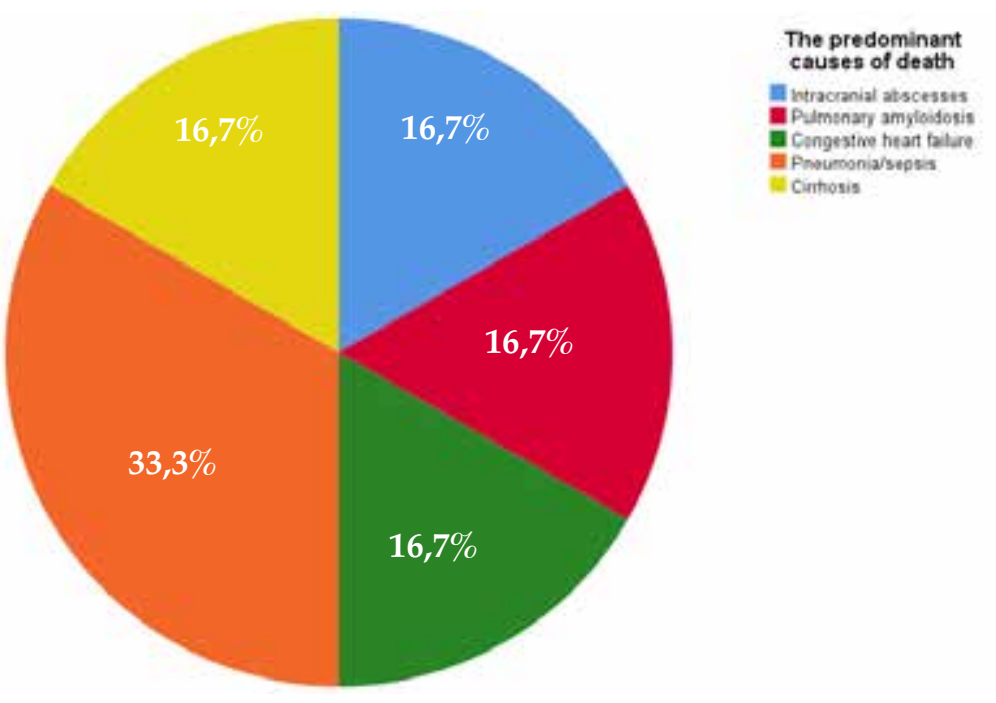




\section{DISCUSSION}

Common variable immunodeficiency is the most common symptomatic primary immunodeficiency in adults (1). In addition to frequent and recurrent respiratory tract infections, patients may present with disorders with immune dysregulation. While infection-related mortality rates have been decreasing owing to immunoglobulin replacement therapy in this patient group, complications of other systems and malignancies have become a significant cause of mortality. In our study, the overall survival rate of the patients was found to be $88 \%$. Furthermore, a lower platelet count was determined to be an independent risk factor for mortality.

B cell defects characterize CVID, and poor vaccine response makes the patients vulnerable to respiratory tract infections with encapsulated bacteria. A study of 224 CVID patients by Quinti et al. (12) reported respiratory tract infections as the most prominent clinical problem both at diagnosis and during the follow-up time. In a 2007 study by Aghamohammadi et al. (5), they reported that the most common cause of mortality in CVID patients was respiratory insufficiency and that the 20-year mortality rate of the patients was $27 \%$. In a study by Gathmann et al. (10), the most common clinical presentation in CVID patients was reported to be pneumonia at a rate of $32 \%$. The fact that our patients' most common clinical presentation was pneumonia and upper respiratory tract infections is consistent with the literature.

Mortality in the CVID patient group is higher compared to the normal population. Although infection-related mortality rates have been reduced with immunoglobulin replacement therapy, mortality due to malignancies and organ complications are still problematic. In a study by Cunningham-Rundles et al. (7), the 20-year mortality rate was reported as 36\% in male CVID patients and 33\% in female CVID patients. In a study by Resnick et al. (9) the mortality rate during a 40-year follow-up time was found to be $19.6 \%$. The median age of death was reported as 44 years in females and 42 years in males. In another study, Quinti et al. (13) reported mortality during a 40year follow-up time as $19.5 \%$ and the median age of death as 54 years, and the primary cause of mortality was reported to be chronic pulmonary diseases (30\%). In our current study, the 10-year mortality rate was determined to be $12 \%$ (with an overall survival rate of $88 \%$ ). The most common cause of mortality, however, was pneumonia and pneumonia-related sepsis. Although our mortality rate was supposed to be lower due to both smaller population and relatively shorter follow-up time of our study compared to aforementioned studies, the fact that our study was recently conducted, therefore, reduction of difficulties in availability of the patients to immunoglobulin replacement therapy and appropriate antibiotics, and advancements in diagnosis and treatment may have lowered the mortality rates.

Immune dysregulation is an important complication in CVID patients. Autoimmunity is a sign of immune dysregulation, and autoimmune complications may be the presenting symptom in $25 \%$ of the patients (14). The coexistence of immunodeficiency with autoimmunity may be considered paradoxical. Because there are poor responses to pathogens and vaccines in CVID and immunoglobulin levels are low; however, autoantibody reproduction may be increased simultaneously. Selftolerance is impaired due to the presence of autoreactive $B$ and T cells. Disorders of innate and adaptive immunities may lead to abnormal B cell clones and the secretion of abnormal cytokines. All these associations may lead to the coexistence of immunodeficiency with autoimmune conditions (15). The most common autoimmune complications in CVID are cytopenias, particularly immune thrombocytopenia (16). In the USIDNET (The United States Immunodeficiency Network) registry system, patients with autoimmune cytopenias have been determined to be associated with non-infectious complications, including lymphoproliferation and granulomatous disorders enteropathy, lymphoma, and interstitial pulmonary diseases (16). In another study, reduced platelet count and switched memory $B$ cell ratio was reported to be a risk factor for bronchiectasis (17). Fisher et al. (18) reported that autoimmune conditions negatively affect the overall survival rate. In a study of 334 CVID patients, Gathmann et al. (10) reported lymphoid malignancies and autoimmunity as independent risk factors for mortality. In a study by Cunningham-Rundles et al. (11), autoimmune cytopenias were associated with increased morbidity and mortality. Also, in our study, reduced platelet count was determined to be an independent risk factor for mortality. These findings suggest that the reduction in platelet count may be an indirect indicator of increased immune dysregulation. As reduced platelet count due to increased immune dysregulation may be associated with other factors causing mortality, primarily lymphoproliferation, 
granulomatous disorders, and chronic pulmonary diseases, reduced platelet count may be associated with mortality.

In conclusion, infections and infection-related sepsis remain to be a significant cause of mortality in CVID patients. Although survival rates in CVID patients are better than before, mortality rates are still higher than in the normal population. Clinicians dealing with this patient group should remember that immune dysregulation and low platelet count are independent risk factors for mortality, and they should particularly follow up patients with low platelet count closely.

\section{Declarations}

The authors received no financial support for the research and/or authorship of this article. There is no conflict of interest.

The study protocol was approved by the Necmettin Erbakan University Meram Medical Faculty Ethics Committee (Date: 03.04.2020 - No: 2020/2401). This study was performed according to the ethical standards laid down Declaration of Helsinki and its later amendments. The authors carried out no animal or human studies for this article.

\section{REFERENCES}

1. Sullivan KE, Puck JM, Notarangelo LD, Fuleihan $R$, Caulder $T$, Wang C, et al. USIDNET: a strategy to build a community of clinical immunologists. J Clin Immunol. 2014;34(4):428-35.

2. Bonilla FA, Barlan I, Chapel H, Costa-Carvalho BT, CunninghamRundles C, de la Morena MT, et al. International Consensus Document (ICON): Common Variable Immunodeficiency Disorders. J Allergy Clin Immunol Pract. 2016;4(1):38-59.

3. Picard C, Bobby Gaspar H, Al-Herz W, Bousfiha A, Casanova JL, Chatila T, et al. International Union of Immunological Societies: 2017 Primary Immunodeficiency Diseases Committee Report on Inborn Errors of Immunity. J Clin Immunol. 2018;38(1):96-128.

4. Chapel H, Lucas M, Lee M, Bjorkander J, Webster D, Grimbacher B, et al. Common variable immunodeficiency disorders: division into distinct clinical phenotypes. Blood. 2008;112(2):277-86.

5. Aghamohammadi A, Pouladi N, Parvaneh N, Yeganeh M, Movahedi $\mathrm{M}$, Gharagolou $\mathrm{M}$, et al. mortality and morbidity in common variable immunodeficiency. J Trop Pediatr. 2007;53(1):32-8

6. Cunningham-Rundles $\mathrm{C}$. How I treat common variable immune deficiency. Blood. 2010;116(1):7-15.

7. Cunningham-Rundles C, Bodian C. Common variable immunodeficiency: clinical and immunological features of 248 patients. Clin Immunol. 1999;92(1):34-48.
8. Kokron CM, Errante PR, Barros MT, Baracho GV, Camargo MM, Kalil J, et al. Clinical and laboratory aspects of common variable immunodeficiency. An Acad Bras Cienc. 2004;76(4):707-26.

9. Resnick ES, Moshier EL, Godbold JH, Cunningham-Rundles C. Morbidity and mortality in common variable immune deficiency over 4 decades. Blood. 2012;119(7):1650-7.

10. Gathmann B, Mahlaoui N, Ceredih, Gerard L, Oksenhendler E, Warnatz K, et al. Clinical picture and treatment of 2212 patients with common variable immunodeficiency. J Allergy Clin Immunol. 2014;134(1):116-26

11. Feuille EJ, Anooshiravani N, Sullivan KE, Fuleihan RL, CunninghamRundles C. Autoimmune Cytopenias and Associated Conditions in CVID: a Report From the USIDNET Registry. J Clin Immunol. 2018;38(1):28-34

12. Quinti I, Soresina A, Spadaro G, Martino S, Donnanno S, Agostini C, et al. Long-term follow-up and outcome of a large cohort of patients with common variable immunodeficiency. J Clin Immunol. 2007;27(3):30816.

13. Quinti I, Agostini C, Tabolli S, Brunetti G, Cinetto F, Pecoraro A, et al. Malignancies are the major cause of death in patients with adult onset common variable immunodeficiency. Blood. 2012;120(9):1953-4.

14. Boileau J, Mouillot G, Gerard L, Carmagnat M, Rabian C, Oksenhendler E, et al. Autoimmunity in common variable immunodeficiency: correlation with lymphocyte phenotype in the French DEFI study. J Autoimmun. 2011;36(1):25-32.

15. Patuzzo G, Barbieri A, Tinazzi E, Veneri D, Argentino G, Moretta F, et al. Autoimmunity and infection in common variable immunodeficiency (CVID). Autoimmun Rev. 2016;15(9):877-82.

16. Agarwal S, Cunningham-Rundles C. Autoimmunity in common variable immunodeficiency. Ann Allergy Asthma Immunol. 2019;123(5):454-60.

17. Aytekin G, Çölkesen F, Yıldız E, Arslan S. Risk Factors of Bronchiectasis in Adult Patients with Common Variable Immunodeficiency. Asthma Allergy Immunol. 2019;17:160-5.

18. Fischer A, Provot J, Jais JP, Alcais A, Mahlaoui N, members of the CFPIDsg. Autoimmune and inflammatory manifestations occur frequently in patients with primary immunodeficiencies. J Allergy Clin Immunol. 2017;140(5):1388-93 e8. 